'Sección Hematología Hospital Clínico de la Universidad de Chile. Santiago, Chile.

${ }^{2}$ Sección Genética Hospital Clínico de la Universidad de Chile. Santiago, Chile.

aprograma de formación de especialista en Hematología. Escuela de Postgrado. Facultad de Medicina. Universidad de Chile.

Trabajo no recibió financiamiento. Los autores declaran no tener conflictos de interés.

Recibido el 15 de enero de 2018, aceptado el 5 de septiembre de

Correspondencia a: Dr. Guillermo Conte

Departamento de Medicina Interna, Sección Hematología, Cuarto Piso Sector D. Santos Dumont 999, Independencia. Santiago, Chile. gfconte@gmail.com

\section{Trombocitopenia hereditaria relacionada a gen $M Y H-9$ : Primera familia reportada en Chile con diagnóstico molecular. Caso clínico}

\author{
GUILLERMO CONTE ${ }^{1}$, MIGUEL LÓPEZ $^{\text {a }}$, PABLO ALARCÓN²
}

\section{Hereditary thrombocytopenia associated with a mutation in the $M Y H-9$ gene. Report of one case}

We report a 51-year-old female who had a first episode of thrombocytopenia at 23 years of age during a pregnancy. At the age of fifty, a hysterectomy was indicated due to a metrorrhagia: a platelet count of 21,000/ul was detected. She was treated with eltrombopag with a good response. The family history of the patient revealed the presence of thrombocytopenia in several family members. Suspecting a hereditary thrombocytopenia, a genetic study revealed a mutation in the MYH-9 gene. This mutation can be suspected when there is a family history of thrombocytopenia with autosomal dominant inheritance, macrothrombocytopenia and in this particular case, due to the response to thrombopoietin receptor agonist, eltrombopag.

(Rev Med Chile 2018; 146: 1074-1078)

Key words: Genetic Diseases, Inborn; Thrombocytopenia; Human Genetics.
L as trombocitopenias hereditarias $(\mathrm{TH})$ son de baja frecuencia. Existe escasa información de prevalencia. El país donde más se han estudiado estas enfermedades es Italia, con una prevalencia de 2,7 x 100.000. No hay información en Chile. Dado el avance de las técnicas moleculares de secuenciación, se han logrado identificar 32 genes que explican 33 tipos de TH conocidas hasta la fecha. Estos genes afectados están involucrados en la proliferación, maduración, formación de gránulos y migración de los megacariocitos ${ }^{1}$. Una clasificación práctica y de rápida orientación diagnóstica de $\mathrm{TH}$ se basa en el tamaño de las plaquetas $^{2}$. En su diagnóstico debe considerarse, además de la trombocitopenia, el tamaño de la plaqueta, tipo de herencia, gen comprometido y otras características. Se detallan las de mayor importancia en la Tabla $1^{1-3}$.

En su mayoría, las $\mathrm{TH}$ se asocian a tenden- cia hemorrágica que se manifiesta en la niñez. La ausencia de manifestaciones de púrpura o hemorragia determina que este diagnóstico sea más tardío, ya sea en la adolescencia o adultez ${ }^{4}$, confundiéndose con las de origen inmune. Por esta razón, la mayoría de los pacientes con $\mathrm{TH}$ no diagnosticadas reciben tratamientos como corticoides, anticuerpos monoclonales, gammaglobulina endovenosa y algunos llegan hasta la esplenectomía, sin respuesta.

\section{Caso clínico}

Mujer de 51 años sin historia de púrpura ni sangrado de mucosas, asintomática, que en ocasión de su primer embarazo, a los 23 años, se constató una trombocitopenia de 35.000/uL. Evolucionó con un parto vaginal normal, sin complicaciones hemorrágicas. Se consideró púrpura 
Trombocitopenia hereditaria MYH9 - G. Conte et al

Tabla 1. Trombocitopenias hereditarias con su tipo de herencia, gen asociado y algunas características

\begin{tabular}{|c|c|c|c|}
\hline Enfermedad & Tipo de herencia & Gen & Características \\
\hline \multicolumn{4}{|l|}{ Plaquetas grandes } \\
\hline TH relacionada a gen $M Y H-9$ & $\begin{array}{l}\text { Autosómica } \\
\text { dominante }\end{array}$ & MYH-9 (22q12-13) & $\begin{array}{l}\text { Plaquetas gigantes, inclusiones en neu- } \\
\text { trófilos tipo cuerpos de Döhle, nefropa- } \\
\text { tía, sordera }\end{array}$ \\
\hline $\begin{array}{l}\text { Bernard Soulier bi y } \\
\text { monoalélico }\end{array}$ & Autosómico recesivo & $\begin{array}{l}\text { GP1BA (17p13), GPIBB } \\
(22 q 11), \text { GP9 (3q21) }\end{array}$ & Plaquetas gigantes, macroplaquetas \\
\hline $\begin{array}{l}\text { Trombocitopenia } \\
\text { Paris-Trousseau }\end{array}$ & $\begin{array}{l}\text { Autosómico } \\
\text { dominante }\end{array}$ & $\begin{array}{l}\text { Grandes deleciones } \\
\text { (11q23-ter) }\end{array}$ & $\begin{array}{l}\text { Alteraciones faciales, defectos cardíacos, } \\
\text { retraso del desarrollo sicomotor y otras }\end{array}$ \\
\hline Síndrome de plaqueta gris & Autosómico recesivo & NBEAL2 (3p21.1) & $\begin{array}{l}\text { Plaquetas grandes hipo y agranulares, } \\
\text { esplenomegalia, mielofibrosis }\end{array}$ \\
\hline $\begin{array}{l}\text { Enfermedad relacionada a } \\
\text { mutaciones GATA1 }\end{array}$ & Ligada al X & GATA1 (Xp11) & $\begin{array}{l}\text { Anemia hemolítica, posible porfiria eri- } \\
\text { tropoyética congénita }\end{array}$ \\
\hline \multicolumn{4}{|l|}{ Plaquetas tamaño normal } \\
\hline $\begin{array}{l}\text { Trombocitopenia } \\
\text { Amegacariocítca congénita }\end{array}$ & Autosómica recesiva & c-MPL (1p34) & $\begin{array}{l}\text { Trombocitopenia y falla medular pro- } \\
\text { gresiva }\end{array}$ \\
\hline $\begin{array}{l}\text { Trombocitopenia con } \\
\text { ausencia de radio }\end{array}$ & Autosómica recesiva & RBM8A (1q21.1) & $\begin{array}{l}\text { El recuento de plaquetas va aumen- } \\
\text { tando con el paso del tiempo, aplasia } \\
\text { bilateral de radio }\end{array}$ \\
\hline $\begin{array}{l}\text { Trombocitopenia familiar } \\
\text { con tendencia a la leucemia } \\
\text { mieloide aguda }\end{array}$ & $\begin{array}{l}\text { Autosómico } \\
\text { dominante }\end{array}$ & CBFA2 (21q22) & $\begin{array}{l}\text { Desarrollo de leucemia mieloide aguda } \\
\text { en el } 40 \% \text { de los pacientes }\end{array}$ \\
\hline $\begin{array}{l}\text { Trombocitopenia relacionada } \\
\text { a mutación ANKRD26 }\end{array}$ & $\begin{array}{l}\text { Autosómico } \\
\text { dominante }\end{array}$ & ANKRD26 (10p2) & Tendencia a leucemia aguda \\
\hline $\begin{array}{l}\text { Trombocitopenia } \\
\text { Amegacariocítica sinostosis } \\
\text { radio-ulnar }\end{array}$ & $\begin{array}{l}\text { Autosómico } \\
\text { dominante }\end{array}$ & HOXA11 (7p15-14) & $\begin{array}{l}\text { Megacarciocitos disminuidos, desarrollo } \\
\text { de falla medular, sinostosis radio-ulnar y } \\
\text { otros defectos }\end{array}$ \\
\hline \multicolumn{4}{|l|}{ Plaquetas pequeñas } \\
\hline Síndrome de Wiscott-Aldrich & Ligada al X & WAS (Xp11) & Inmunodeficiencia severa \\
\hline Trombocitopenia ligada al X & Ligada al X & WAS (Xp11) & Sin inmunodeficiencia \\
\hline
\end{tabular}

trombocitopénico inmune con recuento fluctuante de plaquetas $(3.000$ a $65.000 / \mathrm{uL})$, sin responder a tratamiento esteroidal ni gammaglobulina. A los 50 años, a raíz de una metrorragia prolongada, se indicó histerectomía, con un recuento de plaquetas de 21.000/uL. En abril de 2016 se indicó eltrombopag $50 \mathrm{mg}$ día vía oral, alcanzando un recuento de $130.000 / \mathrm{uL}$ de plaquetas al mes. En mayo de 2016 se realizó histerectomía total sin complicaciones (Figura 1).

Evolucionó asintomática, pero con trombocitopenia, que en enero de 2017 era de 3.000/uL, trasladándose al Hospital de la Universidad de Chile para tratamiento con anticuerpo monoclonal antiCD20 por su carácter inmune.
A su ingreso, el 23 de febrero de 2017, se constató una historia familiar de trombocitopenia en sus dos hijos, una hermana, una prima y varios sobrinos afectados (Figura 2).

Al examen no presentaba púrpura u otras alteraciones hemorrágicas. El frotis de sangre periférica demostró macroplaquetas gigantes, similar al tamaño de un glóbulo rojo e inclusiones citoplasmáticas en leucocitos tipo cuerpos de Döhle (Figura 3).

La biopsia de médula ósea mostró una celularidad de $45 \%$ con normalidad de serie eritroide y granulocítica y megacariocitos hiperlobados. Cariotipo normal 46,XX. El estudio de coagulación mostraba una protrombina en $102 \%$ y un TTPK 


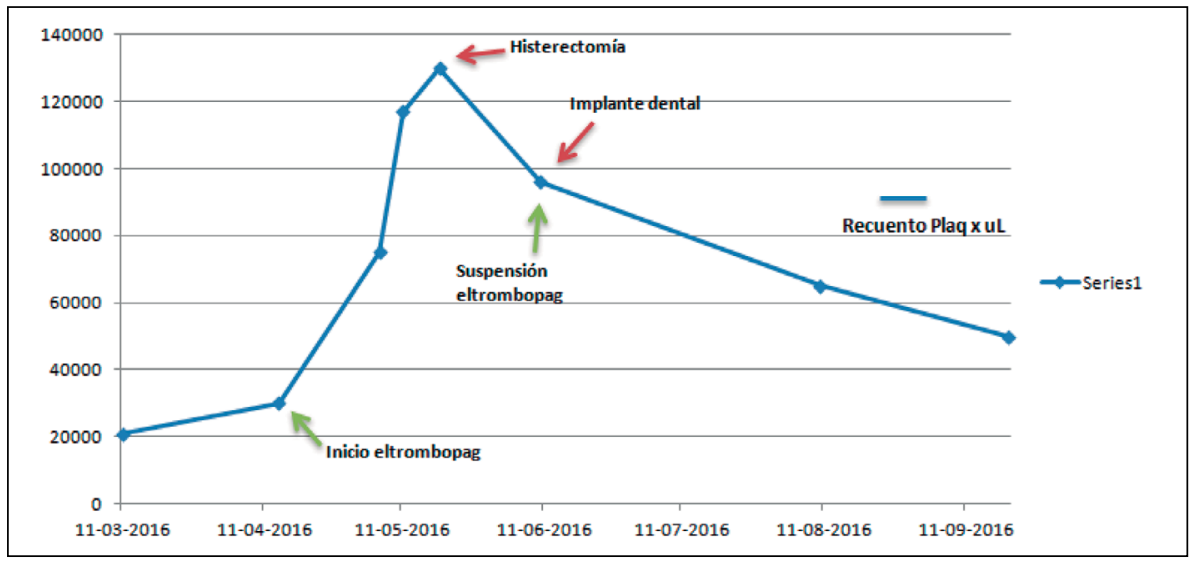

Figura 1. Recuento de plaquetas en relación a uso de eltrombopag.

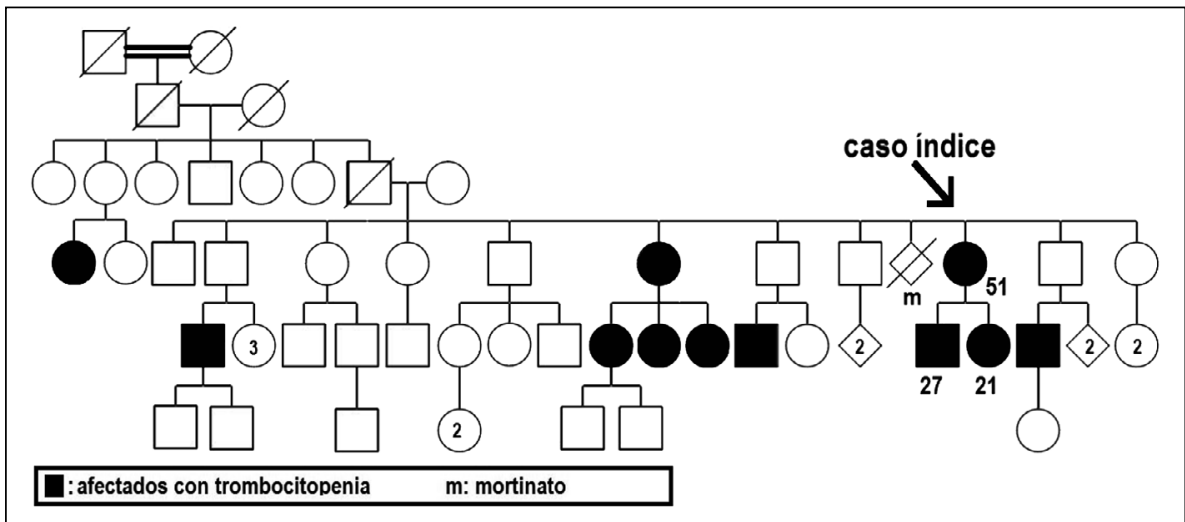

Figura 2. Genograma de la familia que muestra los sujetos afectados con trombocitopenia.

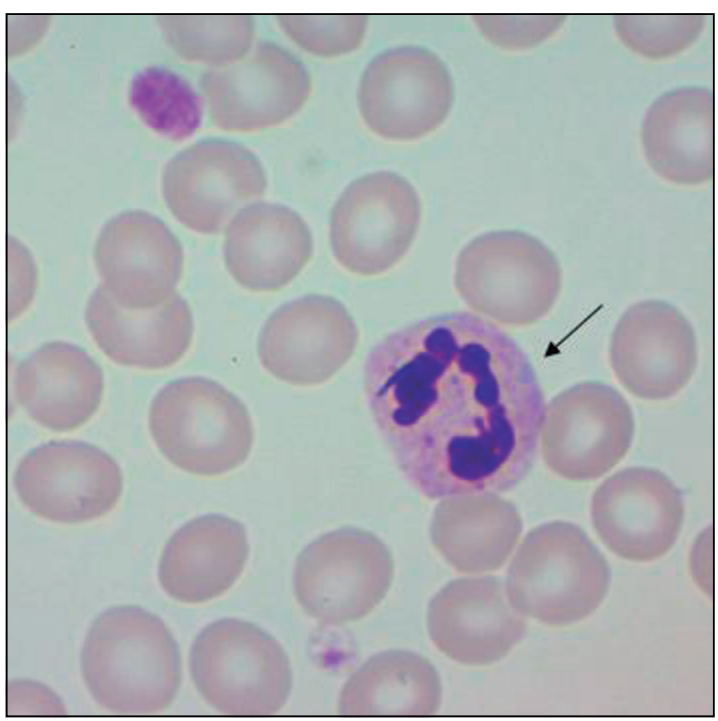

Figura 3. Foto de sangre periférica del caso índice que muestra plaquetas gigantes del tamaño de un glóbulo rojo e inclusiones tipo cuerpos Döhle (flecha) en neutrófilo. Tinción May-Grünwald Giemsa. en 25 segundos, normales. Los antecedentes mencionados y la respuesta a eltrombopag plantea el diagnóstico de una TH relacionada a mutaciones del gen $M Y H-9$. El análisis genético mediante secuenciación exómica por Next Generation Sequencing (Laboratorio CENTOGENE, Alemania) demostró en el gen $\mathrm{MYH}-9$ la variante patogénica c.4270G >A p (Asp 1424Asn) en heterocigosis. Esta variante se encuentra en el exón 31 y ya había sido descrita por Kunishima et al, 20015. Se realizó estudio de ambos hijos con trombocitopenia (hombre de 27 años y mujer de 21 años), mediante secuenciación de Sanger del exón 31, encontrándose en ambos la variante en heterocigosis.

\section{Discusión}

La TH relacionada a mutaciones del gen MYH-9 es una de las formas más frecuentes de $\mathrm{TH}^{2}$. El gen $M Y H-9$ codifica para la proteína 
miosina-IIA que forma parte del citoesqueleto de los megacariocitos. Durante la megacariopoiesis, los megacariocitos migran desde el nicho osteoblástico al nicho sinusoidal y una vez maduros liberan las proplaquetas al lumen sinusoidal. Estudios in vitro e in vivo en ratas con modelos de mutación del gen $\mathrm{MYH}-9$ demostraron que los progenitores megacariocíticos en esta enfermedad expresan menos miosina-IIA y, de esta manera, las proplaquetas se liberan de manera prematura al intersticio medular y no al lumen sinusoidal, lo cual explica la trombocitopenia periférica ${ }^{6,7}$. Además, se ha observado que la alteración de la miosina-IIA lleva a disfunción de la migración de los megacariocitos desde el nicho osteoblástico al nicho sinusoidal ${ }^{8}$. Al año 2012 se habían descrito 220 familias con esta patología ${ }^{1}$. Las TH relacionadas a mutaciones del gen $M Y H-9$ se caracterizan por trombocitopenia con plaquetas gigantes e inclusiones en los leucocitos tipo cuerpos de Döhle. Estas inclusiones corresponden a acúmulos de la proteína mutada de la miosina-IIA ${ }^{9}$. Las plaquetas tienen una función normal ${ }^{2,10}$.

Las mutaciones del gen $M Y H-9$ se manifiestan clínicamente con epistaxis, equimosis y metrorragias. Las hemorragias intracraneales son raras. Es frecuente que los pacientes sean asintomáticos y el hallazgo de macrotrombocitopenia es lo que hace sospechar esta enfermedad. Sin embargo, en 35\% de los casos las mutaciones son de novo por lo cual no tienen antecedentes familiares.

Algunos pacientes desarrollan, con el paso del tiempo, nefropatía proteinúrica, sordera y cataratas preseniles, dependiendo del tipo de mutación que afecte al gen $M Y H-9^{11,12}$.

Plaquetas: Se observa un aumento del tamaño de las plaquetas desde el nacimiento. Los contadores automatizados confunden a las plaquetas gigantes con glóbulos rojos, dando recuentos de plaquetas más bajo de lo real. La agregación plaquetaria con $\mathrm{ADP}$, colágeno y ristocetina es normal. El aspirado y biopsia medular muestran megacariocitos en proporción y morfología normal.

Leucocitos: Los frotis de sangre periférica muestran inclusiones tipo cuerpos de Döhle, observándose en $40-80 \%$ de los pacientes y corresponden a agregados de miosina-IIA ${ }^{9}$.

Los estudios de Pecci, $2008^{11}$ y de Dong, $2005^{12}$ demostraron que las variantes que afectan al dominio principal desarrollan, con el paso del tiempo y en su mayoría, nefropatía, cataratas preseniles y alteraciones sensorioneurales auditivas. Las variantes del dominio cola evolucionan solo con alteraciones hematológicas. Particularmente, se ha observado que los pacientes con sustituciones de aminoácidos en el dominio principal evolucionan con falla renal y sordera antes de los 40 años ${ }^{10}$.

Pecci et al, el año $2010^{13}$, mostraron que el agonista de trombopoyetina, eltrombopag, logró que de 12 pacientes con mutaciones del $\mathrm{MYH}-9$ con recuento de plaquetas basal menor a 50.000/ uL, alcanzaran, en 11 de ellos, recuentos sobre $50.000 /$ uL y que 7 de los 12 pacientes lograran recuentos sobre $100.000 / \mathrm{uL}$ entre las 3 y 6 semanas. En caso de necesidad urgente de aumento de plaquetas, como urgencias quirúrgicas o procedimientos invasivos, la transfusión de plaquetas es la alternativa.

Las TH son enfermedades de baja frecuencia, pero que pueden ser sospechadas desde el punto de vista clínico. La revisión de la literatura en nuestro país no demostró publicaciones al respecto. Nos parece de importancia la presentación de este caso y la revisión de este tipo de patologías para hacer más accesible la sospecha diagnóstica y demostrar que en la actualidad se puede llegar a determinar con exactitud la alteración genética subyacente.

En los últimos años han salido reportes donde pacientes con $\mathrm{TH}$ relacionadas a mutaciones de ANKRD26 desarrollan con el paso del tiempo morfología displásica de la médula ósea, llevando a catalogar a los pacientes como portadores de síndrome mielodisplásicos ${ }^{14}$.

Finalmente, desde el punto de vista del médico general, debe destacarse que una anamnesis completa, con antecedentes familiares, sumado a la revisión de la morfología de la sangre periférica, permite una sospecha de este tipo de enfermedades, cuya confirmación es genética.

\section{Referencias}

1. Noris P, Pecci A. Hereditary thrombocytopenias: a growing list of disorders. Hematology Am Soc Hematol Educ Program 2017; 1: 385-99.

2. Balduini C, Savoia A. Genetics of familial forms of thrombocytopenia. Human Genetics 2012; 131 (12): 1821-32.

3. Greinacher A, Pecci A, Kunishima S, Althaus K, Nurden $\mathrm{P}$, Balduini C, et al. Diagnosis of inherited platelet disorders on a blood smear: a tool to facilitate worldwide 
diagnosis of platelet disorders. J Thromb Haemost 2017; 15: 1511-21.

4. Balduini C, Savoia A, Seri M. Inherited thrombocytopenias frequently diagnosed in adults. J Thromb Haemost 2013; 11 (6): 1006-19.

5. Kunishima S, Matsushita T, Kojima T, Amemiya N, Choi, Y, Hosaka N, et al. Identification of six novel MYH9 mutations and genotype-phenotype relationships in autosomal dominant macrothrombocytopenia with leukocyte inclusions. J Hum Genet 2001; 46 (12): 722-9.

6. Chen Z, Naveiras O, Balduini A, Mammoto A, Conti M, Adelstein R, et al. The May-Hegglin anomaly gene MYH9 is a negative regulator of platelet biogenesis modulated by the Rho-ROCK pathway. Blood 2007; 110 (1): 171-9.

7. Pecci A, Malara A, Badalucco S, Bozzi V, Torti M, Balduini C. Megakaryocytes of patients with MYH9-related thrombocytopenia present an altered proplatelet formation. Thromb Haemost 2009; 102 (1): 90-6.

8. Pecci A, Bozzi V, Panza E, Barozzi S, Gruppi C, Seri $\mathrm{M}$, et al. Mutations responsible for MYH9-related thrombocytopenia impair SDF-1-driven migration of megakaryoblastic cells. Thromb Haemost 2011; 106 (4): 693-704.

9. Savoia A, De Rocco D, Panza E, Bozzi V, Scandellari R,
Loffredo G, et al. Heavy chain myosin 9-related disease (MYH9-RD): neutrophil inclusions of myosin-9 as a pathognomonic sign of the disorder. Thromb Haemost 2010; 103 (4): 826-32.

10. Balduini C, Pecci A, Savoia A. Recent advances in the understanding and management of MYH9-related inherited thrombocytopenias. Br J Haematol 2011; 154 (2): 161-74.

11. Pecci A, Panza E, Pujol-Moix N, Klersy C, Di Bari F, Bozzi V, et al. Position of nonmuscle myosin heavy chain IIA (NMMHC-IIA) mutations predicts the natural history of MYH9-related disease. Human Mutation 2008; 29 (3): 409-17.

12. Dong F, Li S, Pujol-Moix N, Luban N, Shin S, Seo J, et al. Genotype-phenotype correlation in MYH9-related thrombocytopenia. Br J Haematol 2005; 130 (4): 620-7.

13. Pecci A, Gresele P, Klersy C, Savoia A, Noris P, Fierro $\mathrm{T}$, et al. Eltrombopag for the treatment of the inherited thrombocytopenia deriving from MYH9 mutations. Blood 2010; 116 (26): 5832-7.

14. Zaninetti C, Santini V, Tiniakou M, Barozzi S, Savoia A, Pecci A. Inherited thrombocytopenia caused by ANKRD26 mutations misdiagnosed and treated as myelodysplastic syndrome: report on two cases. J Throm Haemost 2017; 15 (12): 2388-92. 